\begin{tabular}{|c|l|}
\hline Title & Semimartingales from the Fokker-Planck Equation \\
\hline Author(s) & Mikami, Toshio \\
\hline Citation & $\begin{array}{l}\text { A pplied Mathematics and Optimization, 53/2), 209-219 } \\
\text { https://doi.org/10.1007/300245-005-0844-z }\end{array}$ \\
\hline Issue Date & 2006-03 \\
\hline Doc URL & http://hdl.handle.net/2115/5781 \\
\hline Rights & The original publication is available at www.springerlink.com \\
\hline Type & article (author version) \\
\hline File Information & AM053-2.pdf \\
\hline
\end{tabular}

Instructions for use 


\title{
Semimartingales from the Fokker-Planck equation
}

\author{
Dedicated to Professor Wendell H. Fleming \\ on the occasion of his seventy seventh birthday \\ Toshio Mikami* \\ Hokkaido University
}

August 31, 2005

\begin{abstract}
We show the existence of a semimartingale of which one-dimensional marginal distributions are given by the solution of the Fokker-Planck equation with the $p$-th integrable drift vector $(p>1)$.
\end{abstract}

AMS (MOS) SUBJECT CLASSIFICATION NUMBERS: 93E20

A shortened version of the title: Semimartingales from the FP equation

\section{Introduction.}

Let $\mathcal{M}_{1}\left(\mathbf{R}^{d}\right)$ denote the complete separable metric space, with a weak topology, of Borel probability measures on $\mathbf{R}^{d}(d \geq 1)$.

Let $b:[0,1] \times \mathbf{R}^{d} \mapsto \mathbf{R}^{d}$ be measurable and $\left\{P_{t}(d x)\right\}_{0 \leq t \leq 1}, \subset \mathcal{M}_{1}\left(\mathbf{R}^{d}\right)$, satisfy the following Fokker-Planck equation: for $f \in C_{b}^{1,2}\left([0,1] \times \mathbf{R}^{d}\right)$ and $t \in[0,1]$,

*Department of Mathematics, Hokkaido University, Sapporo 060-0810, Japan; mikami@math.sci.hokudai.ac.jp; phone \& fax no. 81/11/706/3444; partially supported by the Grant-in-Aid for Scientific Research, No. 15340047, 15340051 and 16654031, JSPS. 


$$
\begin{aligned}
& \int_{\mathbf{R}^{d}} f(t, x) P_{t}(d x)-\int_{\mathbf{R}^{d}} f(0, x) P_{0}(d x) \\
= & \int_{0}^{t} d s \int_{\mathbf{R}^{d}}\left(\frac{\partial f(s, x)}{\partial s}+\frac{1}{2} \triangle f(s, x)+<b(s, x), D_{x} f(s, x)>\right) P_{s}(d x),
\end{aligned}
$$

where $\triangle:=\sum_{i=1}^{d} \partial^{2} / \partial x_{i}^{2}, D_{x}:=\left(\partial / \partial x_{i}\right)_{i=1}^{d}$, and $<\cdot, \cdot>$ denotes the inner product in $\mathbf{R}^{d}$.

Inspired by Born's probabilistic interpretation of a solution to Schrödinger's equation, Nelson proposed the problem of the construction of a diffusion process $\{X(t)\}_{0 \leq t \leq 1}$ for which the following holds (see [20]):

$$
\begin{aligned}
X(t) & =X(0)+\int_{0}^{t} b(s, X(s)) d s+W(t) \quad(t \in[0,1]), \\
P(X(t) \in d x) & =P_{t}(d x) \quad(t \in[0,1]),
\end{aligned}
$$

where $\{W(t)\}_{0 \leq t \leq 1}$ is a $\sigma[X(s): 0 \leq s \leq t]$-Wiener process.

The first result was given by Carlen [2] (see also [23]). It was generalized, by Mikami [12], to the case where the second order differential operator has a variable coefficient. The further generalization and almost complete resolution was made by Cattiaux and Léonard [3-6] (see also [1, 13-15] for the related topics). But in these papers, they assumed that

$$
\int_{0}^{1} d t \int_{\mathbf{R}^{d}}|b(t, x)|^{2} P_{t}(d x)<\infty
$$

for some $b$ for which (1.1) holds. This is called the finite energy condition for $\left\{P_{t}(d x)\right\}_{0 \leq t \leq 1}$.

Remark 1.1 It is known that $b$ is not unique for $\left\{P_{t}(d x)\right\}_{0 \leq t \leq 1}$ in (1.1) (see [12] or [3-6]).

In this paper we consider Nelson's problem under a weaker assumption than (1.4): there exists $p>1$ such that

$$
\int_{0}^{1} d t \int_{\mathbf{R}^{d}}|b(t, x)|^{p} P_{t}(d x)<\infty
$$

for some $b$ for which (1.1) holds. We call (1.5) the generalized finite energy condition for $\left\{P_{t}(d x)\right\}_{0 \leq t \leq 1}$. 
Let $L(t, x ; u):[0,1] \times \mathbf{R}^{d} \times \mathbf{R}^{d} \mapsto[0, \infty)$ be continuous and be convex in $u$. Let $\mathcal{A}$ denote the set of all $\mathbf{R}^{d}$-valued, continuous semimartingales $\{X(t)\}_{0 \leq t \leq 1}$ on a complete filtered probability space such that there exists a Borel measurable $\beta_{X}:[0,1] \times C([0,1]) \mapsto \mathbf{R}^{d}$ for which

(i) $\omega \mapsto \beta_{X}(t, \omega)$ is $\mathcal{B}(C([0, t]))_{+}$-measurable for all $t \in[0,1]$, where $\mathcal{B}(C([0, t]))$ denotes the Borel $\sigma$-field of $C([0, t])$ and $\mathcal{B}(C([0, t]))_{+}$denotes the left hand side limit of $t \mapsto \mathcal{B}(C([0, t]))$,

(ii) $\left\{W_{X}(t):=X(t)-X(0)-\int_{0}^{t} \beta_{X}(s, X) d s\right\}_{0 \leq t \leq 1}$ is a $\sigma[X(s): 0 \leq s \leq t]$ Wiener process.

For $P_{0}$ and $P_{1} \in \mathcal{M}_{1}\left(\mathbf{R}^{d}\right)$, put

$$
\begin{gathered}
V\left(P_{0}, P_{1}\right):=\inf \left\{E\left[\int_{0}^{1} L\left(t, X(t) ; \beta_{X}(t, X)\right) d t\right] \mid\right. \\
\left.P X(t)^{-1}=P_{t}(t=0,1), X \in \mathcal{A}\right\} \\
:=\inf \left\{\int_{0}^{1} \int_{\mathbf{R}^{d}} L(t, x ; b(t, x)) P(t, d x) d t \mid P(t, d x)=P_{t}(d x)(t=0,1),\right. \\
\left.\quad\{P(t, d x)\}_{0 \leq t \leq 1} \subset \mathcal{M}_{1}\left(\mathbf{R}^{d}\right),(b(t, x), P(t, d x)) \text { satisfies }(1.1)\right\} .
\end{gathered}
$$

In [12] where $u \mapsto L$ is quadratic, we proved and used the following:

$$
V\left(P_{0}, P_{1}\right)=v\left(P_{0}, P_{1}\right) .
$$

Remark 1.2 As a typical case, when $L=|u|^{2}$, the minimizer of $V\left(P_{0}, P_{1}\right)$ is known to be the h-path process for the space-time Brownian motion (see [7, 18] and the references therein). It is known that its zero-noise limit exists and is the unique minimizer of Monge's problem (see [16, 19]).

In this paper we prove (1.8) for a more general function $L$ by the duality theorem for $V$. To make the point clearer, we describe [18] briefly. For $P_{0}$ and $P_{1} \in \mathcal{M}_{1}\left(\mathbf{R}^{d}\right)$, put

$$
\mathcal{V}\left(P_{0}, P_{1}\right):=\sup \left\{\int_{\mathbf{R}^{d}} \varphi(1, y) P_{1}(d y)-\int_{\mathbf{R}^{d}} \varphi(0, x) P_{0}(d x)\right\},
$$


where the supremum is taken over all classical solutions $\varphi$ to the following Hamilton-Jacobi-Bellman equation:

$$
\begin{aligned}
\frac{\partial \varphi(t, x)}{\partial t}+\frac{1}{2} \Delta \varphi(t, x)+H\left(t, x ; D_{x} \varphi(t, x)\right) & =0\left((t, x) \in(0,1) \times \mathbf{R}^{d}\right)(1.1 \\
\varphi(1, \cdot) & \in C_{b}^{\infty}\left(\mathbf{R}^{d}\right)
\end{aligned}
$$

(see Lemma 3.1). Here for $(t, x, z) \in[0,1] \times \mathbf{R}^{d} \times \mathbf{R}^{d}$,

$$
H(t, x ; z):=\sup _{u \in \mathbf{R}^{d}}\{<z, u>-L(t, x ; u)\} .
$$

The following was proved in [18] and is called the duality theorem for the stochastic optimal control problem (1.6).

Theorem 1.1 (Duality Theorem) Suppose that (A.1)-(A.4) in section 2 hold. Then for any $P_{0}$ and $P_{1} \in \mathcal{M}_{1}\left(\mathbf{R}^{d}\right)$,

$$
V\left(P_{0}, P_{1}\right)=\mathcal{V}\left(P_{0}, P_{1}\right)(\in[0, \infty]) .
$$

Suppose in addition that $V\left(P_{0}, P_{1}\right)$ is finite. Then $V\left(P_{0}, P_{1}\right)$ has a minimizer and for any minimizer $\{X(t)\}_{0 \leq t \leq 1}$ of $V\left(P_{0}, P_{1}\right)$,

$$
\beta_{X}(t, X)=b_{X}(t, X(t)):=E\left[\beta_{X}(t, X) \mid(t, X(t))\right] .
$$

Remark 1.3 (1.12) can be considered as a counterpart in the stochastic optimal control theory of the duality theorem in the Monge-Kantorovich problem (see [10, 17, 21, 22] and the references therein).

Using a similar result to (1.8) on small time intervals $\subset[0,1]$, we prove that for $\mathbf{P}:=\left\{P_{t}(d x)\right\}_{0 \leq t \leq 1} \subset \mathcal{M}_{1}\left(\mathbf{R}^{d}\right)$,

$$
\mathbf{V}(\mathbf{P})=\mathbf{v}(\mathbf{P})
$$

where

$\mathbf{V}(\mathbf{P}):=\inf \left\{E\left[\int_{0}^{1} L\left(t, X(t) ; \beta_{X}(t, X)\right) d t\right] \mid P X(t)^{-1}=P_{t}(0 \leq t \leq 1), X \in \mathcal{A}\right\}$ 


$$
\mathbf{v}(\mathbf{P}):=\inf \left\{\int_{0}^{1} d t \int_{\mathbf{R}^{d}} L(t, x ; b(t, x)) P_{t}(d x) \mid b \text { satisfies }(1.1)\right\} .
$$

In particular, the existence of a minimizer of $\mathbf{V}(\mathbf{P})$ implies that of a semimartingale for which (1.2)-(1.3) hold. When $p=2$ in (1.5), this semimartingale is Markovian. But we do not know if it is also true even when $1<p<2$. This is our future problem.

In section 2 we state our result which will be proved in section 4 . Technical lemmas are given in section 3.

I would like to dedicate this paper to Professor Wendell H. Fleming on the occasion of his seventy seventh birthday. I would like to thank him for his constant encouragement since I was a student of his.

\section{Main result.}

In this section we state our result.

We state assumptions on $L$.

(A.1). There exists $p>1$ such that

$$
\liminf _{|u| \rightarrow \infty} \frac{\inf \left\{L(t, x ; u):(t, x) \in[0,1] \times \mathbf{R}^{d}\right\}}{|u|^{p}}>0 .
$$

(A.2).

$$
\Delta L\left(\varepsilon_{1}, \varepsilon_{2}\right):=\sup \frac{L(t, x ; u)-L(s, y ; u)}{1+L(s, y ; u)} \rightarrow 0 \quad \text { as } \varepsilon_{1}, \varepsilon_{2} \rightarrow 0,
$$

where the supremum is taken over all $(t, x)$ and $(s, y), \in[0,1] \times \mathbf{R}^{d}$, for which $|t-s| \leq \varepsilon_{1},|x-y|<\varepsilon_{2}$ and all $u \in \mathbf{R}^{d}$.

(A.3). (i) $L(t, x ; u) \in C^{3}\left([0,1] \times \mathbf{R}^{d} \times \mathbf{R}^{d}:[0, \infty)\right)$,

(ii) $D_{u}^{2} L(t, x ; u)$ is positive definite for all $(t, x, u) \in[0,1] \times \mathbf{R}^{d} \times \mathbf{R}^{d}$,

(iii) $\sup \left\{L(t, x ; o):(t, x) \in[0,1] \times \mathbf{R}^{d}\right\}$ is finite,

(iv) $\left|D_{x} L(t, x ; u)\right| /(1+L(t, x ; u))$ is bounded,

(v) $\sup \left\{\left|D_{u} L(t, x ; u)\right|:(t, x) \in[0,1] \times \mathbf{R}^{d},|u| \leq R\right\}$ is finite for all $R>0$.

(A.4). (i) $\Delta L(0, \infty)$ is finite, or (ii) $p=2$ in (A.1).

Remark 2.1 (i). (A.3, ii) implies that $L(t, x ; u)$ is strictly convex in $u$. (ii). $\left(1+|u|^{2}\right)^{p / 2}(p>1)$ satisfies (A.1)-(A.3) and $(A .4, i)$. 
We state that (1.8) holds.

Theorem 2.1 Suppose that (A.1)-(A.4) hold. Then for any $P_{0}$ and $P_{1} \in$ $\mathcal{M}_{1}\left(\mathbf{R}^{d}\right)$

$$
V\left(P_{0}, P_{1}\right)=v\left(P_{0}, P_{1}\right)(\in[0, \infty]) .
$$

The following is our main result (see (1.15)-(1.16) for notations).

Theorem 2.2 Suppose that (A.1)-(A.4) hold. Then

(i) for any $\mathbf{P}:=\left\{P_{t}(d x)\right\}_{0 \leq t \leq 1} \subset \mathcal{M}_{1}\left(\mathbf{R}^{d}\right)$,

$$
\mathbf{V}(\mathbf{P})=\mathbf{v}(\mathbf{P})(\in[0, \infty]) .
$$

(ii) For any $\mathbf{P}:=\left\{P_{t}(d x)\right\}_{0 \leq t \leq 1}, \subset \mathcal{M}_{1}\left(\mathbf{R}^{d}\right)$, for which $\mathbf{v}(\mathbf{P})$ is finite, there exist a unique minimizer $b_{o}(t, x)$ of $\mathbf{v}(\mathbf{P})$ and a minimizer $X \in \mathcal{A}$, of $\mathbf{V}(\mathbf{P})$. In particular, for any minimizer $X \in \mathcal{A}$, of $\mathbf{V}(\mathbf{P})$,

$$
\beta_{X}(t, X)=b_{o}(t, X(t))
$$

and (1.2)-(1.3) with $b=b_{o}$ hold.

Remark 2.2 If $\mathbf{v}(\mathbf{P})$ is finite, then the generalized finite energy condition (1.5) holds from (A.1).

\section{Lemmas.}

In this section we give technical lemmas.

In the same way as to $\mathcal{A}$, we define the set of semimartingales $\mathcal{A}_{t}$ in $C([t, 1])$. We recall the following result.

Lemma 3.1 ([8, p. 210, Remark 11.2] ) Suppose that (A.1) and (A.3) hold. Then for any $f \in C_{b}^{\infty}\left(\mathbf{R}^{d}\right)$, the HJB equation (1.10) with $\varphi(1, \cdot)=f$ has a unique solution $\varphi \in C^{1,2}\left([0,1] \times \mathbf{R}^{d}\right) \cap C_{b}^{0,1}\left([0,1] \times \mathbf{R}^{d}\right)$, which can be written as follows:

$$
\begin{aligned}
\varphi(t, x)=\sup _{X \in \mathcal{A}_{t}}\{ & E[f(X(1)) \mid X(t)=x] \\
& \left.-E\left[\int_{t}^{1} L\left(s, X(s) ; \beta_{X}(s, X)\right) d s \mid X(t)=x\right]\right\},
\end{aligned}
$$


where for the maximizer $X \in \mathcal{A}_{t}$, the following holds:

$$
\beta_{X}(s, X)=D_{z} H\left(s, X(s) ; D_{x} \varphi(s, X(s))\right) .
$$

Fix $P_{0} \in \mathcal{M}_{1}\left(\mathbf{R}^{d}\right)$. For $f \in C_{b}\left(\mathbf{R}^{d}\right)$, put

$$
\begin{aligned}
V^{*}(f) & :=\sup _{P \in \mathcal{M}_{1}\left(\mathbf{R}^{d}\right)}\left\{\int_{\mathbf{R}^{d}} f(x) P(d x)-V\left(P_{0}, P\right)\right\}, \\
v^{*}(f) & :=\sup _{P \in \mathcal{M}_{1}\left(\mathbf{R}^{d}\right)}\left\{\int_{\mathbf{R}^{d}} f(x) P(d x)-v\left(P_{0}, P\right)\right\} .
\end{aligned}
$$

The following lemma plays a crucial role in the proof of Theorem 2.1.

Lemma 3.2 (i) Suppose that $\left(A .3, i\right.$, ii) hold. Then for any $Q_{0}$ and $Q_{1} \in$ $\mathcal{M}_{1}\left(\mathbf{R}^{d}\right)$,

$$
V\left(Q_{0}, Q_{1}\right) \geq v\left(Q_{0}, Q_{1}\right)
$$

(ii) Suppose in addition that (A.1) and (A.3) hold. Then for any $f \in$ $C_{b}^{\infty}\left(\mathbf{R}^{d}\right)$,

$$
V^{*}(f) \geq v^{*}(f)
$$

(Proof) We first prove (i). For $X \in \mathcal{A}$ for which $E\left[\int_{0}^{1} L\left(t, X(t) ; \beta_{X}(t, X)\right) d t\right]$ is finite and for which $P X(t)^{-1}=Q_{t}(t=0,1),\left(b_{X}(t, x), P(X(t) \in d x)\right)$ satisfies (1.1) with $\left(b(t, x), P_{t}(d x)\right)=\left(b_{X}(t, x), P(X(t) \in d x)\right)$ (see (1.13) for notation). Indeed, for any $f \in C_{b}^{1,2}\left([0,1] \times \mathbf{R}^{d}\right)$ and $t \in[0,1]$, by Itô's formula,

$$
\begin{aligned}
& \int_{\mathbf{R}^{d}} f(t, x) P(X(t) \in d x)-\int_{\mathbf{R}^{d}} f(0, x) P(X(0) \in d x) \\
= & E[f(t, X(t))-f(0, X(0))] \\
= & \int_{0}^{t} d s E\left[\frac{\partial f(s, X(s))}{\partial s}+\frac{1}{2} \triangle f(s, X(s))+<\beta_{X}(s, X), D_{x} f(s, X(s))>\right] \\
= & \int_{0}^{t} d s E\left[\frac{\partial f(s, X(s))}{\partial s}+\frac{1}{2} \triangle f(s, X(s))+<b_{X}(s, X(s)), D_{x} f(s, X(s))>\right] \\
= & \int_{0}^{t} d s \int_{\mathbf{R}^{d}}\left(\frac{\partial f(s, x)}{\partial s}+\frac{1}{2} \triangle f(s, x)+<b_{X}(s, x), D_{x} f(s, x)>\right) P(X(s) \in d x) .
\end{aligned}
$$


Hence, from Remark 2.1, (i), by Jensen's inequality,

$$
\begin{aligned}
& E\left[\int_{0}^{1} L\left(t, X(t) ; \beta_{X}(t, X)\right) d t\right] \\
\geq & E\left[\int_{0}^{1} L\left(t, X(t) ; b_{X}(t, X(t))\right) d t\right] \\
= & \int_{0}^{1} d t \int_{\mathbf{R}^{d}} L\left(t, x ; b_{X}(t, x)\right) P(X(t) \in d x) \geq v\left(Q_{0}, Q_{1}\right) .
\end{aligned}
$$

Next we prove (ii). For $\varphi$ in (3.1) and $\{(b(t, x), P(t, d x))\}_{0 \leq t \leq 1}$ for which $\{P(t, d x)\}_{0 \leq t \leq 1} \subset \mathcal{M}_{1}\left(\mathbf{R}^{d}\right)$ and (1.1) with $P(0, d x)=P_{0}$ holds,

$$
\int_{\mathbf{R}^{d}} f(x) P(1, d x)-\int_{\mathbf{R}^{d}} \varphi(0, x) P_{0}(d x) \leq \int_{0}^{1} d t \int_{\mathbf{R}^{d}} L(t, x ; b(t, x)) P(t, d x) .
$$

Indeed, take $\psi \in C_{o}^{\infty}\left(\mathbf{R}^{d}:[0, \infty)\right)$ for which $\psi(x)=1(|x| \leq 1)$ and $\psi(x)=0$ $(|x| \geq 2)$, and put $\psi_{R}(x):=\psi(x / R)$ for $R>0$. Then from (1.1),

$$
\begin{gathered}
\int_{\mathbf{R}^{d}} \psi_{R}(x) f(x) P(1, d x)-\int_{\mathbf{R}^{d}} \psi_{R}(x) \varphi(0, x) P(0, d x) \\
=\int_{0}^{1} d t \int_{\mathbf{R}^{d}} \psi_{R}(x)\left[\frac{\partial \varphi(t, x)}{\partial t}+\frac{1}{2} \triangle \varphi(t, x)+<b(t, x), D_{x} \varphi(t, x)>\right] P(t, d x) \\
+\int_{0}^{1} d t \int_{\mathbf{R}^{d}}\left[<D_{x} \psi_{R}(x), D_{x} \varphi(t, x)>+\frac{1}{2} \triangle \psi_{R}(x) \varphi(t, x)\right. \\
\left.+<b(t, x), D_{x} \psi_{R}(x)>\varphi(t, x)\right] P(t, d x) .
\end{gathered}
$$

Let $R \rightarrow \infty$. Then we obtain (3.8) from (1.10), (A.1) and Lemma 3.1.

Lemma 3.1 and (3.8) implies (ii). Indeed,

$$
\begin{gathered}
v^{*}(f)=\sup \left\{\int_{\mathbf{R}^{d}} f(x) P(1, d x)-\int_{0}^{1} d t \int_{\mathbf{R}^{d}} L(t, x ; b(t, x)) P(t, d x) \mid\right. \\
P(0, d x)=P_{0}(d x),\{P(t, d x)\}_{0 \leq t \leq 1} \subset \mathcal{M}_{1}\left(\mathbf{R}^{d}\right) \\
(b(t, x), P(t, d x)) \text { satisfies }(1.1) \cdot\} \\
\leq \int_{\mathbf{R}^{d}} \varphi(0, x) P_{0}(d x) \quad(\text { from }(3.8))
\end{gathered}
$$




$$
\begin{aligned}
& =\sup \left\{E\left[f(X(1))-\int_{0}^{1} L\left(t, X(t) ; \beta_{X}(t, X)\right) d t\right] \mid\right. \\
& \left.\quad P X(0)^{-1}=P_{0}, X \in \mathcal{A}\right\} \quad(\text { from Lemma 3.1) } \\
& =V^{*}(f) . \square
\end{aligned}
$$

Let $\left(\Omega, \mathbf{B},\left\{\mathbf{B}_{t}\right\}_{t \geq 0}, P\right)$ be a complete filtered probability space, $X_{o}$ be a $\left(\mathbf{B}_{0}\right)$-adapted random variable, and $\{W(t)\}_{t \geq 0}$ denote a d-dimensional $\left(\mathbf{B}_{t}\right)$ Wiener process for which $W(0)=o$ (see e.g., [11]). For a $\mathbf{R}^{d}$-valued, $\left(\mathbf{B}_{t}\right)$ progressively measurable stochastic process $\{u(t)\}_{0 \leq t \leq 1}$, put

$$
X^{u}(t)=X_{o}+\int_{0}^{t} u(s) d s+W(t) \quad(t \in[0,1]) .
$$

Then the following is known.

Lemma 3.3 Suppose that $E\left[\int_{0}^{1}|u(t)| d t\right]$ is finite. Then $\left\{X^{u}(t)\right\}_{0 \leq t \leq 1} \in \mathcal{A}$ and

$$
\beta_{X^{u}}\left(t, X^{u}\right)=E\left[u(t) \mid X^{u}(s), 0 \leq s \leq t\right]
$$

(see [11, p. 270]). Besides, by Jensen's inequality,

$$
E\left[\int_{0}^{1} L\left(t, X^{u}(t) ; u(t)\right) d t\right] \geq E\left[\int_{0}^{1} L\left(t, X^{u}(t) ; \beta_{X^{u}}\left(t, X^{u}\right)\right) d t\right] .
$$

For $\mathbf{P}:=\left\{P_{t}(d x)\right\}_{0 \leq t \leq 1} \subset \mathcal{M}_{1}\left(\mathbf{R}^{d}\right)$ and $n \geq 1$, put

$$
\begin{gathered}
V_{n}(\mathbf{P}):=\inf \left\{E\left[\int_{0}^{1} L\left(t, X(t) ; \beta_{X}(t, X)\right) d t\right] \mid\right. \\
\left.P X(t)^{-1}=P_{t}\left(t=\frac{i}{2^{n}}, i=0, \cdots, 2^{n}\right), X \in \mathcal{A}\right\} \\
v_{n}(\mathbf{P}):=\inf \left\{\int_{0}^{1} d t \int_{\mathbf{R}^{d}} L(t, x ; b(t, x)) P(t, d x) \mid\right. \\
P(t, d x)=P_{t}(d x)\left(t=\frac{i}{2^{n}}, i=0, \cdots, 2^{n}\right) \\
\left.\{P(t, d x)\}_{0 \leq t \leq 1} \subset \mathcal{M}\left(\mathbf{R}^{d}\right),(b(t, x), P(t, d x)) \text { satisfies }(1.1)\right\}
\end{gathered}
$$

Then we have 
Lemma 3.4 Suppose that (A.1)-(A.4) hold. Then for any $\mathbf{P}:=\left\{P_{t}(d x)\right\}_{0 \leq t \leq 1} \subset$ $\mathcal{M}_{1}\left(\mathbf{R}^{d}\right)$ and $n \geq 1$,

$$
v_{n}(\mathbf{P})=V_{n}(\mathbf{P})
$$

(Proof) For $i=0, \cdots, 2^{n}-1$, put

$$
\begin{aligned}
& V_{n, i}(\mathbf{P}):=\inf \left\{E\left[\int_{0}^{\frac{1}{2^{n}}} L\left(t, X(t) ; \beta_{X}(t, X)\right) d t\right] \mid\right. \\
& \left.P X(t)^{-1}=P_{t+\frac{i}{2^{n}}}\left(t=0, \frac{1}{2^{n}}\right), X \in \mathcal{A}\right\}, \\
& :=\inf \left\{\int_{0}^{\frac{1}{2^{n}}} d t \int_{\mathbf{R}^{d}} L(t, x ; b(t, x)) P(t, d x) \mid\right. \\
& \quad P(t, d x)=P_{t+\frac{i}{2^{n}}}(d x)\left(t=0, \frac{1}{2^{n}}\right),\{P(t, d x)\}_{0 \leq t \leq \frac{1}{2^{n}}} \subset \mathcal{M}\left(\mathbf{R}^{d}\right) \\
& \left.\quad(b(t, x), P(t, d x)) \text { satisfies }(1.1) \text { on }\left[0,1 / 2^{n}\right]\right\}
\end{aligned}
$$

Then, from Theorem 2.1,

$$
v_{n}(\mathbf{P})=\sum_{i=0}^{2^{n}-1} v_{n, i}(\mathbf{P})=\sum_{i=0}^{2^{n}-1} V_{n, i}(\mathbf{P}) .
$$

Since $V_{n}(\mathbf{P}) \geq v_{n}(\mathbf{P})$ from (3.6)-(3.7), we only have to prove the following:

$$
\sum_{i=0}^{2^{n}-1} V_{n, i}(\mathbf{P}) \geq V_{n}(\mathbf{P}) .
$$

Suppose that the left hand side of (3.20) is finite. For $i=0, \cdots 2^{n}-1$, take a minimizer $X_{n, i}$ of $V_{n, i}(\mathbf{P})$ (see Theorem 1.1), and put

$$
P_{n, i}:=P X_{n, i}\left(\cdot-\frac{i}{2^{n}}\right)^{-1} \quad \text { on }\left(C\left(\left[\frac{i}{2^{n}}, \frac{i+1}{2^{n}}\right]: \mathbf{R}^{d}\right), \mathcal{B}\left(C\left(\left[\frac{i}{2^{n}}, \frac{i+1}{2^{n}}\right]: \mathbf{R}^{d}\right)\right)\right),
$$




$$
\begin{aligned}
P_{n}\left(\left.d X\right|_{C\left([0,1]: \mathbf{R}^{d}\right)}\right):= & P_{n, 0}\left(\left.d X\right|_{C\left(\left[0, \frac{1}{2^{n}}\right]: \mathbf{R}^{d}\right)}\right) \\
& \times \prod_{i=1}^{2^{n}-1} P_{n, i}\left(\left.d X\right|_{C\left(\left[\frac{i}{2^{n}}, \frac{i+1}{\left.\left.2^{n}\right]: \mathbf{R}^{d}\right)} \mid\right.\right.} \mid X_{n, i}\left(\frac{i}{2^{n}}\right)=X\left(\frac{i}{2^{n}}\right)\right)
\end{aligned}
$$

on $\left(C\left([0,1]: \mathbf{R}^{d}\right), \mathcal{B}\left(C\left([0,1]: \mathbf{R}^{d}\right)\right)\right)$. Under the completion of this measure, the coordinate process $\left\{X_{n}(t)\right\}_{0 \leq t \leq 1}$ satisfies the following:

$$
X_{n}(t)=X_{n}(0)+\sum_{i=0}^{2^{n}-1} \int_{\min \left(\frac{i}{2^{n}}, t\right)}^{\min \left(\frac{i+1}{2^{n}}, t\right)} b_{n, i}\left(s-\frac{i}{2^{n}}, X_{n}(s)\right) d s+W_{X_{n}}(t) \quad(0 \leq t \leq 1),
$$

where $b_{n, i}$ denotes the drift vector of $X_{n, i}$ (see Theorem 1.1). In particular, $P X_{n}(t)^{-1}=P_{t}\left(t=i / 2^{n}, i=0, \cdots, 2^{n}\right)$, which implies (3.20).

\section{Proofs.}

In this section we prove our results given in section 2 .

When $L=|u|^{2}$, the following proof extremely simplifies that of $[12$, Lemma 2.5].

(Proof of Theorem 2.1). Lemma 3.2, (i) and the following complete the proof:

$$
\begin{aligned}
& v\left(P_{0}, P_{1}\right) \\
\geq & \sup _{f \in C_{b}^{\infty}\left(\mathbf{R}^{d}\right)}\left\{\int_{\mathbf{R}^{d}} f(x) P_{1}(d x)-v^{*}(f)\right\} \quad(\text { from }(3.3)) \\
\geq & \sup _{f \in C_{b}^{\infty}\left(\mathbf{R}^{d}\right)}\left\{\int_{\mathbf{R}^{d}} f(x) P_{1}(d x)-V^{*}(f)\right\} \quad \text { (from Lemma 3.2, (ii)) } \\
= & V\left(P_{0}, P_{1}\right) \quad(\text { from Theorem } 1.1(\text { see }(3.10))) .
\end{aligned}
$$

(Proof of Theorem 2.2). We first prove (i). From (3.6)-(3.7), $\mathbf{V}(\mathbf{P}) \geq \mathbf{v}(\mathbf{P})$. Therefore we only have to show that

$$
\mathbf{v}(\mathbf{P}) \geq \mathbf{V}(\mathbf{P}) .
$$

Suppose that $\mathbf{v}(\mathbf{P})$ is finite. Then, from Lemma 3.4, 


$$
\mathbf{v}(\mathbf{P}) \geq v_{n}(\mathbf{P})=V_{n}(\mathbf{P})
$$

and $X_{n}$ constructed in (3.23) is a minimizer of $V_{n}(\mathbf{P})$.

Let $b_{n}$ denote the drift vector of $\left\{X_{n}(t)\right\}_{0 \leq t \leq 1}$. It is easy to see that $\left\{\left(X_{n}(t), \int_{0}^{t} b_{n}\left(s, X_{n}(s)\right) d s\right): t \in[0,1]\right\}_{n \geq 1}$ is tight in $C\left([0,1]: \mathbf{R}^{2 d}\right)$ from (A.1) (see [23, Theorem 3] or [9]). Take a weakly convergent subsequence $\left\{\left(X_{n_{k}}(t), \int_{0}^{t} b_{n_{k}}\left(s, X_{n_{k}}(s)\right) d s\right): t \in[0,1]\right\}_{k \geq 1}$ such that

$$
\begin{aligned}
& \liminf _{n \rightarrow \infty} E\left[\int_{0}^{1} L\left(t, X_{n}(t) ; b_{n}\left(s, X_{n}(s)\right)\right) d t\right] \\
= & \lim _{k \rightarrow \infty} E\left[\int_{0}^{1} L\left(t, X_{n_{k}}(t) ; b_{n_{k}}\left(s, X_{n_{k}}(s)\right)\right) d t\right] .
\end{aligned}
$$

Let $\{(X(t), A(t))\}_{t \in[0,1]}$ denote the limit of $\left\{\left(X_{n_{k}}(t), \int_{0}^{t} b_{n_{k}}\left(s, X_{n_{k}}(s)\right) d s\right): t \in\right.$ $[0,1]\}_{k \geq 1}$ as $k \rightarrow \infty$. Then $\{X(t)-X(0)-A(t)\}_{t \in[0,1]}$ is a $\sigma[X(s): 0 \leq s \leq t]$ Wiener process and $\{A(t)\}_{t \in[0,1]}$ is absolutely continuous (see [23, Theorem $5]$ or [9]). We can also prove, in the same way as in the proof of [15, (3.17)], the following: from (4.3)-(4.4), (A.2) and (A.3, ii) (see Remark 2.1, (i)),

$$
\begin{aligned}
\mathbf{v}(\mathbf{P}) & \geq \liminf _{n \rightarrow \infty} E\left[\int_{0}^{1} L\left(t, X_{n}(t) ; b_{n}\left(t, X_{n}(t)\right)\right) d t\right] \\
& \geq E\left[\int_{0}^{1} L\left(t, X(t) ; \frac{d A(t)}{d t}\right) d t\right] \\
& \geq \tilde{E}\left[\int_{0}^{1} L\left(t, X(t) ; \beta_{X}(t, X)\right) d t\right] \quad(\text { from Lemma 3.3) } \\
& \geq \mathbf{V}(\mathbf{P}) .
\end{aligned}
$$

Here $\tilde{E}$ denotes the mean value by the completion of $P X(\cdot)^{-1}$ and we used the fact that $P(X(t) \in d x)=P_{t}(d x)$ for all $t \in[0,1]$. Indeed,

$$
\begin{gathered}
P(X(t) \in d x)=\lim _{n \rightarrow \infty} P\left(X\left(\frac{\left[2^{n} t\right]}{2^{n}}\right) \in d x\right) \quad \text { weakly, } \\
P\left(X\left(\frac{\left[2^{n} t\right]}{2^{n}}\right) \in d x\right)=P_{\frac{\left[2^{n} t\right]}{2^{n}}}(d x) \rightarrow P_{t}(d x) \quad \text { as } n \rightarrow \infty \text { weakly. }
\end{gathered}
$$


Next we prove (ii). Suppose that $\mathbf{v}(\mathbf{P})$ is finite. Then (2.2) and (4.5) show the existence of a minimizer $X$ of $\mathbf{V}(\mathbf{P})$. In the same way as in (3.7), Theorem 2.2, (i) and the strict convexity of $u \mapsto L(t, x ; u)$ (see Remark 2.1, (i)) imply that $\beta_{X}(t, X)=b_{X}(t, X(t))$ and $b_{X}(t, x)$ is a minimizer of $\mathbf{v}(\mathbf{P})$.

Let $b_{1}$ and $b_{2}$ be minimizers of $\mathbf{v}(\mathbf{P})$. Then for any $\lambda \in(0,1), \lambda b_{1}(t, x)+$ $(1-\lambda) b_{2}(t, x)$ satisfies (1.1), and

$$
\begin{aligned}
& \mathbf{v}(\mathbf{P}) \\
\leq & \int_{0}^{1} \int_{\mathbf{R}^{d}} L\left(t, x ; \lambda b_{1}(t, x)+(1-\lambda) b_{2}(t, x)\right) P_{t}(d x) \\
\leq & \lambda \int_{0}^{1} \int_{\mathbf{R}^{d}} L\left(t, x ; b_{1}(t, x)\right) P_{t}(d x)+(1-\lambda) \int_{0}^{1} \int_{\mathbf{R}^{d}} L\left(t, x ; b_{2}(t, x)\right) P_{t}(d x) \\
= & \mathbf{v}(\mathbf{P}) .
\end{aligned}
$$

The strict convexity of $u \mapsto L(t, x ; u)$ implies the uniqueness of a minimizer of $\mathbf{v}(\mathbf{P})$.

\section{References}

[1] Blumenthal RM, Corson HH (1972) On continuous collections of measures, in: Le Cam, L., Neyman, J., Scott, E.L. (Eds.), Proc. 6th Berkeley Sympos. Math. Statist. Probab. 2, Berkeley 1970/1971. Univ. California press, Berkeley, pp. 33-40

[2] Carlen EA (1984) Conservative diffusions. Commun Math Phys 94:293315

[3] Cattiaux P, Léonard C (1994) Minimization of the Kullback information of diffusion processes. Ann Inst H Poincar Probab Statist 30:83-132

[4] Cattiaux P, Léonard C (1995) Correction to: "Minimization of the Kullback information of diffusion processes" [Ann. Inst. H. Poincar Probab. Statist. 30 (1994), no. 1, 83-132]. Ann Inst H Poincar Probab Statist 31:705-707

[5] Cattiaux P, Léonard C (1995) Large deviations and Nelson processes. Forum Math 7:95-115 
[6] Cattiaux P, Léonard C (1996) Minimization of the Kullback information for some Markov processes, in: Azema, J., Emery, M., Yor, M. (Eds.), Séminaire de Probabilités, XXX, Lecture Notes in Math., Vol. 1626. Springer-Verlag, Berlin, Heidelberg, New York, Tokyo, pp. 288-311

[7] Doob JL (1984) Classical potential theory and its probabilistic counterpart. Springer-Verlag, Berlin, Heidelberg, New York, Tokyo

[8] Fleming WH, Soner HM (1993) Controlled Markov Processes and Viscosity Solutions. Springer-Verlag, Berlin, Heidelberg, New York, Tokyo

[9] Jacod J, Shiryaev AN (1987) Limit theorems for stochastic processes. Springer-Verlag, Berlin, Heidelberg, New York, Tokyo

[10] Kellerer HG (1984) Duality theorems for marginal problems. Z Wahrsch Verw Gebiete 67:399-432

[11] Liptser RS, Shiryaev AN (1977) Statistics of random processes I. Springer-Verlag, Berlin, Heidelberg, New York, Tokyo

[12] Mikami T (1990) Variational processes from the weak forward equation. Commun Math Phys 135:19-40

[13] Mikami T (1999) Markov marginal problems and their applications to Markov optimal control, in: McEneaney, W. M., Yin, G. G., Zhang, Q. (Eds.), Stochastic Analysis, Control, Optimization and Applications, A Volume in Honor of W. H. Fleming. Birkhäuser, Boston, pp. 457-476

[14] Mikami T (2000) Dynamical systems in the variational formulation of the Fokker-Planck equation by the Wasserstein metric. Appl Math Optim 42:203-227

[15] Mikami T (2002) Optimal control for absolutely continuous stochastic processes and the mass transportation problem. Elect Comm in Probab 7:199-213

[16] Mikami T (2004) Monge's problem with a quadratic cost by the zeronoise limit of $h$-path processes. Probab Theory Related Fields 129:245260 
[17] Mikami T (2004) A Simple Proof of Duality Theorem for MongeKantorovich Problem. To appear in Kodai Math J

[18] Mikami T, Thieullen M (2004) Duality Theorem for Stochastic Optimal Control Problem. Hokkaido University preprint series, \#652

[19] Mikami T, Thieullen M (2005) Optimal Transportation Problem by Stochastic Optimal Control. Hokkaido University preprint series, \#690

[20] Nelson E (1967) Dynamical theories of Brownian motion. Princeton University Press, Princeton

[21] Rachev ST, Rüschendorf L (1998) Mass transportation problems, Vol. I: Theory, Vol. II: Application. Springer-Verlag, Berlin, Heidelberg, New York, Tokyo

[22] Villani C (2003) Topics in Optimal Transportation. Amer Math Soc, Providence.

[23] Zheng WA (1985) Tightness results for laws of diffusion processes application to stochastic mechanics. Ann Inst Henri Poincaré 21:103-124 